\title{
Assessment of Antimicrobial Agents, Analgesics, and Epidermal Growth Factors-Embedded Anti-Adhesive Poly(Lactic-Co-Glycolic Acid) Nanofibrous Membranes: In vitro and in vivo Studies
}

\author{
Kuo-Sheng Liu (D) \\ Ching-Wei Kao ${ }^{2}$ \\ Yuan-Yun Tseng $\mathbb{1 D}^{3}$ \\ Shih-Kuang Chen ${ }^{4}$ \\ Yu-Ting Lin ${ }^{4}$ \\ Chia-Jung $\mathrm{Lu}^{4,5}$ \\ Shih-Jung Liu (D) ${ }^{4,5}$
}

'Department of Thoracic and Cardiovascular Surgery, Linkou Chang

Gung Memorial Hospital, Taoyuan,

Taiwan; ${ }^{2}$ Department of Anesthesiology, Chiayi Chang Gung Memorial Hospital, Chiayi, Taiwan; ${ }^{3}$ Department of Neurosurgery, New Taipei Municipal TuCheng Hospital (Built and Operated by Chang Gung Medical Foundation), New Taipei City, Taiwan; ${ }^{4}$ Department of Mechanical Engineering, Chang Gung University, Taoyuan, Taiwan;

${ }^{5}$ Department of Orthopedic Surgery, Bone and Joint Research Center, Linkou Chang Gung Memorial Hospital, Taoyuan, Taiwan

Correspondence: Shih-Jung Liu Biomaterials Lab, Mechanical Engineering, Chang Gung University, 259, Wen-Hwa

Ist Road, Kwei-Shan, Tao-Yuan 333 Taiwan

Tel +886-3-2II8I66

Fax +886-3-2II8558

Email shihjung@mail.cgu.edu.tw
Background: Postoperative tissue adhesion is a major concern for most surgeons and is a nearly unpreventable complication after abdominal or pelvic surgeries. This study explored the use of sandwich-structured antimicrobial agents, analgesics, and human epidermal growth factor (hEGF)-incorporated anti-adhesive poly(lactic-co-glycolic acid) nanofibrous membranes for surgical wounds.

Materials and Methods: Electrospinning and co-axial electrospinning techniques were utilized in fabricating the membranes. After spinning, the properties of the prepared membranes were assessed. Additionally, high-performance liquid chromatography and enzymelinked immunosorbent assays were utilized in assessing the in vitro and in vivo liberation profiles of the pharmaceuticals and the hEGF from the membranes.

Results: The measured data suggest that the degradable anti-adhesive membranes discharged high levels of vancomycin/ceftazidime, ketorolac, and hEGF in vitro for more than 30, 24, and 27 days, respectively. The in vivo assessment in a rat laparotomy model indicated no adhesion in the peritoneal cavity at 14 days post-operation, demonstrating the anti-adhesive capability of the sandwich-structured nanofibrous membranes. The nanofibers also released effective levels of vancomycin, ceftazidime, and ketorolac for more than 28 days in vivo. Histological examination revealed no adverse effects.

Conclusion: The outcomes of this study implied that the anti-adhesive nanofibers with sustained release of antimicrobial agents, analgesics, and growth factors might offer postoperative pain relief and infection control, as well as promote postoperative healing of surgical wounds.

Keywords: nanofibrous anti-adhesive membrane, poly(lactic-co-glycolic acid), extended release, vancomycin, ceftazidime, ketorolac, hEGF

\section{Introduction}

Postoperative tissue adhesion is a major concern for most surgeons and is a nearly unpreventable complication after abdominal or pelvic surgeries. Adhesions can induce significant post-surgery diseases, such as bowel obstruction, infertility, chronic pelvic pain, and/or abdominal pain. ${ }^{1}$ The incidence of post-surgical intraabdominal adhesions ranges from $50 \%$ to $95 \%$ among women who have undergone 
gynecological surgeries. ${ }^{2}$ Distinct treatment methods have been proposed to reduce post-surgical adhesion, including reducing peritoneal damage through sophisticated surgical techniques, pharmacological blockade of fibrin formation, and minimization of contact between organs by using physical adhesion barriers. ${ }^{2}$ Among these, the use of adhesion barriers is particularly encouraging. ${ }^{3-9}$ The barriers are embeddable substances that reduce abnormal internal adhesions after surgery by physically separating the tissues and organs during the curative process. ${ }^{10}$ Films, gels, or other substances can be used as physical barriers for segregating injured tissues before the suturing of the incision site, and they generally biodegrade at 3 to 7 days after the healing of the tissue surface.

Postoperative wound infection is a common problem that can lead to pain, impaired wound healing, the requirement for prolonged antibiotic use, reoperation, extended hospitalization, and elevated health care costs. ${ }^{11}$ It has been reported that the development of surgical site infection depends on the type of operation and may arise in 5$20 \%$ of operations, leading to 7 to 11 additional postsurgical days of hospitalization and 2- to 11-times higher risk of mortality than in non-infected patients. ${ }^{12}$ Pain that occurs after an operation is a significant concern. Despite the progress in pain management, some patients still experience postoperative pain. ${ }^{13}$ Post-surgical pain can affect a patient's surgical result, comfort, satisfaction from clinic care, as well as directly influence the development of tachycardia, hyperventilation, decrease in alveolar ventilation, conversion to chronic pain, deficient wound healing, and insomnia. ${ }^{14,15}$

Currently, there are few reports on the development of drugs and growth factor-eluting anti-adhesive implants. Chen et al advocated for the use of silver nanoparticles/ ibuprofen-incorporated poly(L-lactide) fibrous films after assessing their anti-infection/anti-adhesion effectiveness. ${ }^{16}$ Kao et al exploited analgesics-incorporated anti-adhesive membranes to offer sustainable elution of lidocaine and ketorolac to relieve postoperative pain. ${ }^{17,18}$ Khan et al ${ }^{19}$ proposed that the laser-mediated surface activation of graphene oxide offers high efficiency for antifungal and antibacterial. Hussein et $\mathrm{al}^{20}$ developed ultrasonicated graphene oxide that provides a good environment for cells involved in bone and skin healing. An ideal antiadhesion implant should possess features such as biocompatibility and biodegradability, easy adherence to the injured surface, vigor on the sleeping surface, and capability for delivery of pharmaceuticals for infection control, pain relief, and healing promotion.

In this study, sandwich-structured vancomycin, ceftazidime, ketorolac, and human epidermal growth factor (hEGF)-embedded anti-adhesive poly(lactic-co-glycolic acid) (PLGA) nanofibrous membranes were used on surgical wounds. Divarvand et al investigated the antiadhesive and anti-inflammatory effects of electrospun nanofibrous membranes made of PLGA, polycaprolactone (PCL), and polyethersulfone (PES) in comparison with the oxidized-regenerated cellulose (Interceed). ${ }^{21}$ Their results show that among the materials, mice receiving PLGA membranes exhibited the least adhesion bands. Both electrospinning and co-axial electrospinning methods were employed in preparing the sandwich-structured nanofibrous membranes. After spinning, the characteristics of the electrospun membranes were assessed. The in vitro and in vivo liberation profiles of the pharmaceuticals and the hEGF from the electrospun nanofibrous membranes were investigated. Additionally, the effectiveness of the antibiotics/analgesic/hEGF-incorporated anti-adhesive nanofibers was evaluated using a rat laparotomy model. Histological assays of the incision sites were also performed.

\section{Materials and Methods Preparation of Sandwich Structured Nanofibrous Membranes}

Sandwich-configuration nanofibrous membranes consisting of a PLGA/ketorolac membrane, a sheath-core structured PLGA/hEGF membrane, and a PLGA/vancomycin/ ceftazidime membrane were prepared using both electrospinning and co-electrospinning devices (see Supporting Information File). The molecular weight of PLGA (50:50) (RG503, Sigma-Aldrich, MO, USA) was 33,000 Da. Other materials, including ketorolac, hEGF, vancomycin hydrochloride, and ceftazidime hydrate, were purchased from Sigma-Aldrich.

To prepare the PLGA/ketorolac nanofibers, PLGA $(1568 \mathrm{mg})$ and ketorolac $(196 \mathrm{mg})$ were mixed with $7 \mathrm{~mL}$ of hexafluoroisopropanol (HFIP) (Sigma-Aldrich). The PLGA/ketorolac mixture was transferred to a syringe equipped with a needle for electrospinning into a nonwoven nanofibrous membrane. The internal diameter of the needle was $0.43 \mathrm{~mm}$. The delivery rate was $0.5 \mathrm{~mL} / \mathrm{h}$, and the voltage applied to the syringe tip was $15 \mathrm{kV}$. The distance from the tip to the collection plate was $12 \mathrm{~cm}$. 
To fabricate the sheath-core structured PLGA/hEGF, PLGA $(1.400 \mathrm{mg})$ was mixed with $4 \mathrm{~mL}$ of HFIP, while hEGF $(0.04 \mathrm{mg})$ was dissolved in $1 \mathrm{~mL}$ of distilled water. After that, the PLGA/hEGF mixtures were transported to two distinct syringes for co-spinning. The internal diameters of the needles for the sheath and core layers were $1.20 \mathrm{~mm}$ and $0.43 \mathrm{~mm}$, respectively. The transport speed of the sheath-layer solution was $0.3 \mathrm{~mL} / \mathrm{h}$, while the dispensing speed for the core-layer solution was $0.1 \mathrm{~mL} / \mathrm{h}$. The direct-current voltage maintained at the syringe tip was $17 \mathrm{kV}$, while the travel length between the syringe tip and the grounded collecting plate was set at $15 \mathrm{~cm}$.

To manufacture the PLGA/vancomycin/ceftazidime nanofibers, PLGA (1568 mg), vancomycin (196 mg), and ceftazidime $(196 \mathrm{mg})$ were mixed with $7 \mathrm{~mL}$ of HFIP (Sigma-Aldrich). The internal diameter of the needle was $1.20 \mathrm{~mm}$. The delivery rate was $0.5 \mathrm{~mL} / \mathrm{h}$, and the voltage applied to the syringe tip was $15 \mathrm{kV}$. The travel distance from the tip to the collection plate was $12 \mathrm{~cm}$.

\section{Scanning Electron Microscope (SEM) Observation}

To estimate the size distribution of the nanofibers, 50 fibers were arbitrarily selected from SEM (JSM-7500F, Tokyo, Japan) images, followed by diameter measurements.

\section{Assessment of Sheath-Core Structured Nanofibers}

The fibrous morphology of the sheath-core structured membranes was assessed using transmission electron microscopy (TEM) (JEOL JEM-2000EXII, Japan).

A Leica TS SP8X laser-scanning confocal microscope (Tokyo, Japan) was employed to confirm the presence of proteins in the sheath/core structured nanofibers, with PLGA as the sheath layer and recombinant enhanced green fluorescent protein (reGFP) as the core. The excitation wavelength for observing the co-axial electrospun nanofibrous membranes was set at $487 \mathrm{~nm}$.

\section{Mechanical Tests}

The mechanical characteristics of the sandwich-configured nanofibrous membranes were evaluated using a tensile test machine (Lloyd, Ametek, USA). The extension rate of the membranes was set to $60 \mathrm{~mm} / \mathrm{min}$.

\section{Wetting Angles}

The wettability of the spun nanofibers was evaluated by measuring their wetting angles (water contact angles) (First Ten Angstroms, Newark, CA, USA). A drop of distilled water was placed on the nanofibers with a $1 \times 1 \mathrm{~cm}$ size to form a dome shape on the surface. The wetting angles were then estimated using a video monitor $(n=3)$.

\section{Fourier Transform Infrared (FTIR) Assay}

To examine the spectra of pure PLGA nanofibers and drug-loaded sandwich-structured PLGA nanofibers, Fourier transform infrared (FTIR) spectroscopy was performed on a Bruker Tensor 27 spectrophotometer. Nanofibrous samples were compressed as $\mathrm{KBr}$ discs and assayed at $400-4000 \mathrm{~cm}^{-1}$. The resolution was set at $4 \mathrm{~cm}^{-1}$ and 32 scans.

\section{In vitro Elution of Nanofibers}

Nanofibrous specimens with a $1 \times 1 \mathrm{~cm}$ size were prepared from the sandwich-configuration membranes and deposited in the assay tubes $(\mathrm{N}=3)$ holding $1 \mathrm{~mL}$ of buffered solution at $37^{\circ} \mathrm{C}$ for $24 \mathrm{~h}$. The mixture was collected and analyzed. The new buffered solution was replaced daily, and the entire procedure was repeated for 30 days.

The elution profiles of ketorolac, vancomycin, and ceftazidime were assessed using HPLC (highperformance liquid chromatography) assay, while the eluted concentrations of hEGF were assessed using an enzyme-linked immunosorbent assay (ELISA).

\section{In vivo Animal Tests}

The in vivo test processes were approved by the Institutional Animal Care and Use Committee of Chang Gung University. All animals were cared for under the supervision of a licensed veterinarian, consistent with the regulations of the National Institute of Health of Taiwan. Twenty-one adult Wistar rats (approximately $250 \mathrm{~g}$ each) were employed for the experiments; 18 animals were used for the in vivo drugs/hEGF concentration analysis, while the other three animals were enrolled as the control $(n=3)$. For the drugs/biomolecules concentration tests, the animals were anesthetized using isoflurane.

A midline laparotomy, approximately $4 \mathrm{~cm}$ in length, was performed in the lower abdomen of each rat. The cecum was identified. The surface of the cecum and the peritoneal wall in correspondence with the cecum were 
scratched with surgical gauze until minimal surface bleeding occurred. The drugs/hEGF nanofibrous membrane $(4 \times$ $2 \mathrm{~cm}$ in dimension) was applied to the surface of the cecum and fixed to the peritoneal wall using two 7-0 polypropylene stitches. The laparotomy incision was then sealed using 3-0 Vicryl sutures. The rats were sacrificed for local tissue sampling at postoperative days $1,3,7,14$, 21, and 28. Blood specimens were also collected via cardiac puncture. Drugs and growth factor concentrations in the specimens were evaluated using HPLC analysis and ELISA, respectively. The peritoneal wall and the cecum that were in direct contact with the nanofibers were sampled for histological examination.

\section{Results}

\section{Assessment of Sandwich-Configuration Nanofibrous Membranes}

Drug/growth factor-loaded sandwich-configuration nanofibrous membranes were satisfactorily manufactured using electrospinning and co-electrospinning processes. Figure 1 shows the SEM images of the electrospun nanofibers and the fiber size distributions. The evaluated diameters for PLGA/ketorolac, PLGA/hEGF, and PLGA/vancomycin/ ceftazidime nanofibers were $263.4 \pm 28.2 \mathrm{~nm}, 747.0 \pm$ $48.9 \mathrm{~nm}$, and $74.5 \pm 4.9 \mathrm{~nm}$, respectively. Figure $2 \mathrm{~A}$ shows the TEM image of the co-electrospun nanofibers, where a sheath-core structure can be observed. Meanwhile, Figure 2B shows the presence of bioactive proteins in the co-axially spun nanofibers, with clear string-like green signals of reGFP.

Figure 3 illustrates the FTIR spectra of the pure PLGA membranes, the drugs, and the hEGF-incorporated PLGA membranes. The novel peaks at $1625 \mathrm{~cm}^{-1}$ and $1575 \mathrm{~cm}^{-1}$ were mainly due to the $\mathrm{N}-\mathrm{H}$ bonds and the $\mathrm{C}=\mathrm{C}$ bonds of the pharmaceuticals, respectively. ${ }^{22}$ The peak at $1790 \mathrm{~cm}^{-1}(\mathrm{C}=\mathrm{O}$ bond) was enhanced in the presence of the drugs. Additionally, the vibration peak close to $3000 \mathrm{~cm}^{-1}$ might result from the $\mathrm{CH}_{3}$ bond enhancement of the embedded drugs. ${ }^{23,24}$ The FTIR spectra assay demonstrated that the pharmaceuticals were successfully embedded in the PLGA membranes.

Figure 4 suggests that the wetting angles of the PLGA/ ketorolac and the PLGA/vancomycin/ceftazidime nanofibers were $70.69^{\circ}$ and $116.49^{\circ}$, respectively. The addition of analgesic greatly enhanced the hydrophilicity of the PLGA nanofibrous membranes. The contact angle of the PLGA/hEGF nanofibers was $133.26^{\circ}$. Since the sheath layer was pure PLGA, the membrane exhibited hydrophobic characteristics.
The experimental outcomes in Figure 5 indicate that the ultimate stress and the maximal strain of the PLGA nanofibers decreased significantly with the addition of the pharmaceuticals and the hEGF.

\section{Liberation Profiles of the Pharmaceuticals and the hEGF from the Sandwich-Configuration Nanofibrous Membranes}

Figure 6 illustrates the in vitro daily and cumulative liberation profiles of the analgesics and antibiotics from the nanofibers. All the drugs exhibited a triphasic liberation feature: a burst release at 1 to 2 days, a second peak discharge at 5 to 10 days, and a steady and progressively decreasing drug elution. Additionally, the spun nanofibrous membranes sustainably eluted ketorolac for 25 days, while the membranes also discharged high levels of vancomycin and ceftazidime (greater than their minimum inhibitory concentrations) for over 30 days in vitro. Conversely, the ELISA assay data in Figure 7 suggest that the sheath-core-structured nanofibers offer sustainable liberation of raised concentrations of hEGF for more than 28 days.

\section{In vivo Animal Study results}

Figure 8 illustrates the in vivo elution profiles of the pharmaceuticals in the tissue and the blood. The concentrations of all the drugs remained significant at the target region for 4 weeks, while the drug levels remained much lower systemically.

Figure 9A shows the implantation of the nanofibrous membranes in rats. No tissue adhesion was observed for 14 days post-implantation (Figure 9B), demonstrating the anti-adhesive capability of the drug-growth factorincorporated nanofibers. Conversely, tissue adhesion was conspicuous in the control group (Figure 9C).

Figure 10 shows the hematoxylin and eosin-stained sections of the cecum wall with and without drug-eluting membrane covering. The histological images of both groups showed interstitial infiltration of inflammatory cells in the submucosa and muscularis externa, indicating surgical trauma.

\section{Discussion}

In this study, we exploited multi-drugs/hEGF-incorporated sandwich-structured anti-adhesive nanofibrous PLGA membranes. Various agents, including films, gels, and other substances, have been used as physical shelters for segregating injured tissues during the healing process. ${ }^{10,11}$ Compared to 

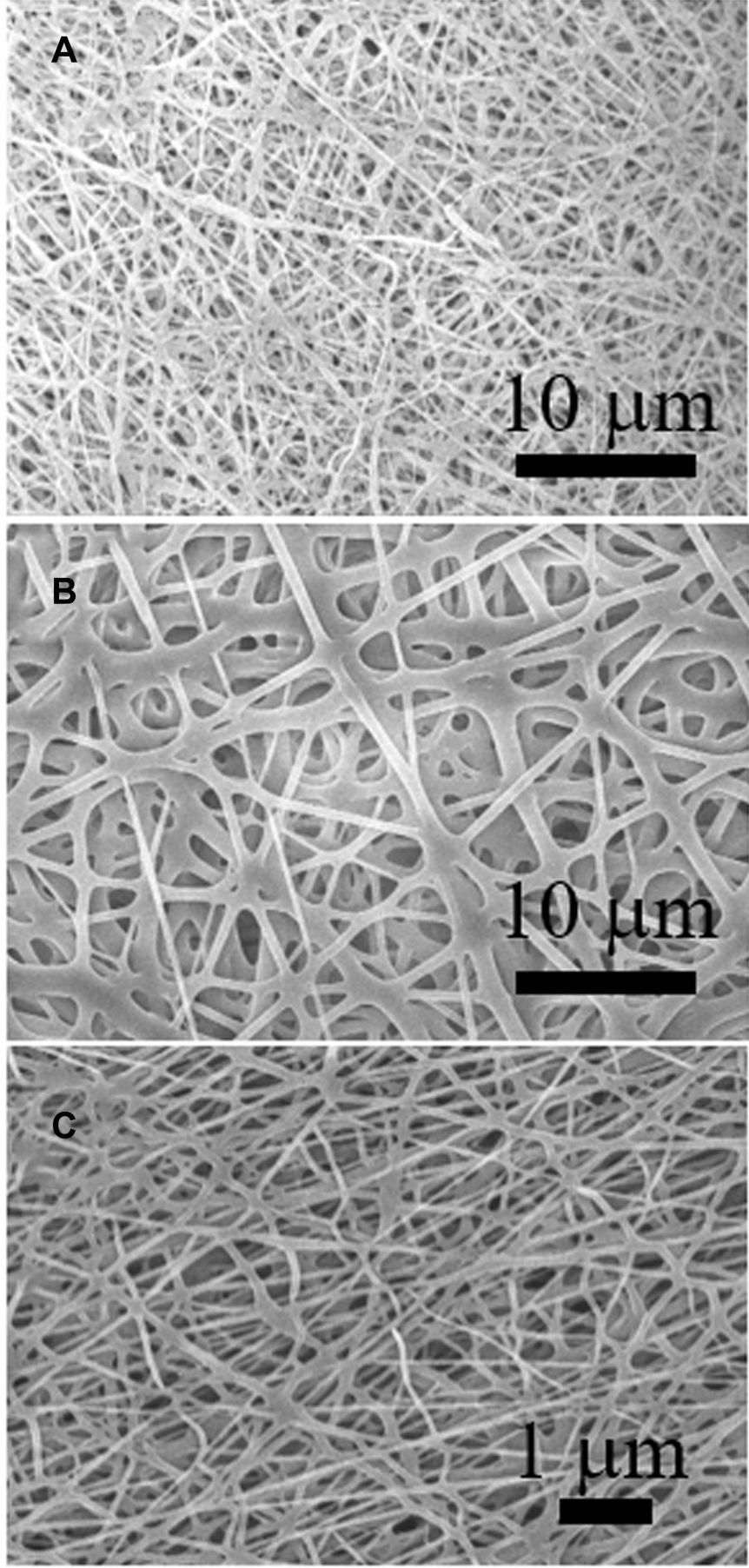
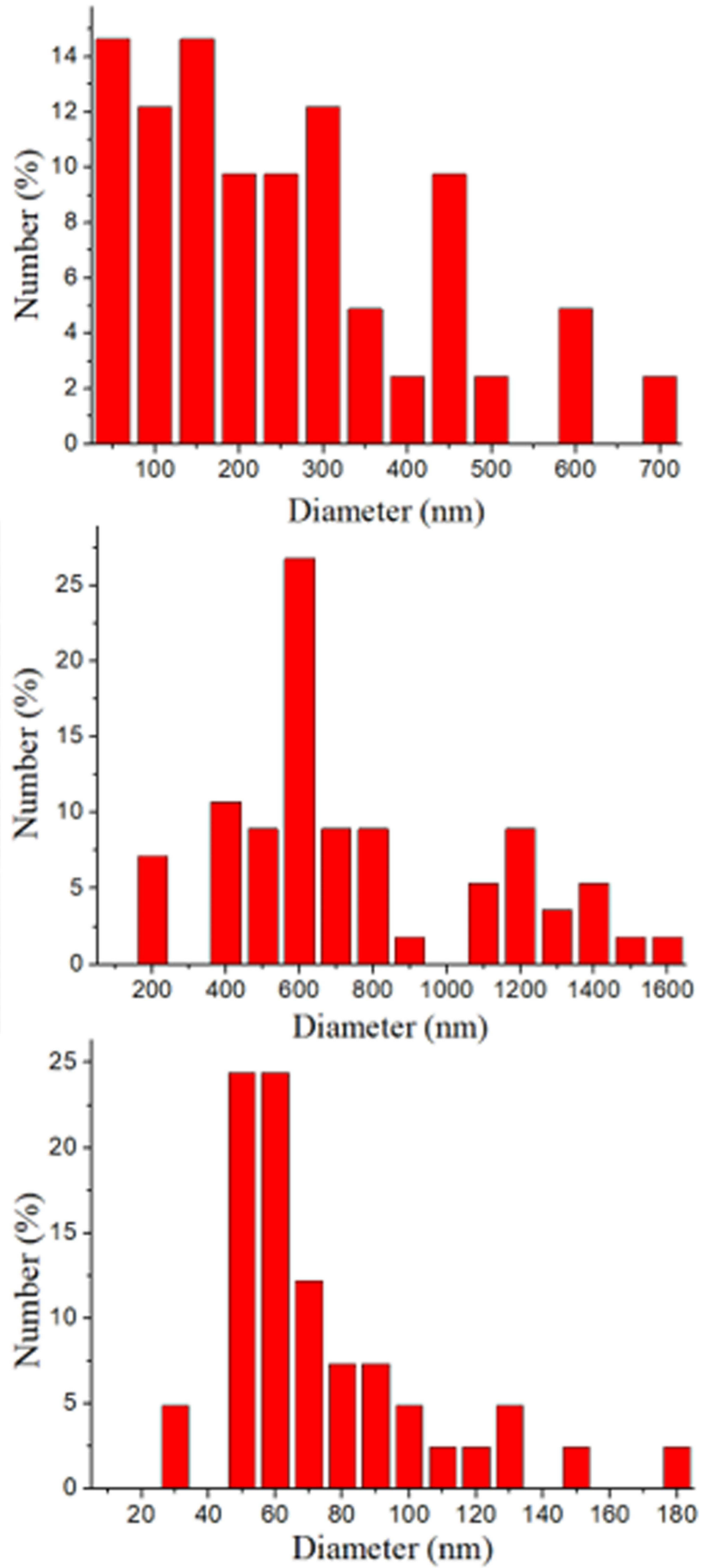

Figure I SEM image and fiber size distribution of (A) PLGA/ketorolac, (B) PLGA/hEGF, and (C) PLGA/vancomycin/ceftazidime nanofibers.

these agents, the tailorable porosity and pore size of nanofibrous membranes allow them to mimic the extracellular matrix (ECM) to promote cell proliferation. ${ }^{25}$ The large surface-to-volume ratio of electrospun nanofibers also offers a considerable number of bioactive agent binding sites, making it a promising candidate for multiple biomedical applications, including anti-adhesive membranes. Additionally, the electrospun nanofibers can be surface-functionalized with optimal mechanical properties that maintain the integrity and match with native ECM. ${ }^{26}$

Among the distinct resorbable polymers, PLGA has been one of the most successful biomaterials for use in controlled drug delivery systems and tissue engineering applications. It is also an FDA-approved degradable polymer that is highly 


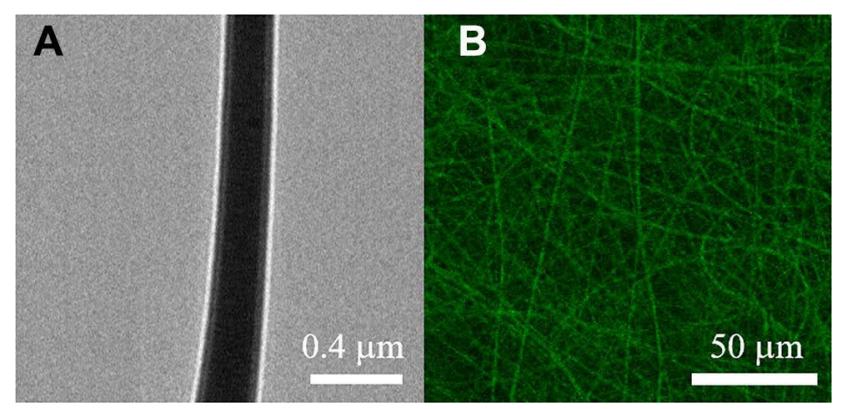

Figure 2 (A) TEM of PLGA/hEGF sheath-core nanofiber, (B) laser scanning confocal microscopy images of reGFP in co-axial electrospun nanofibers.

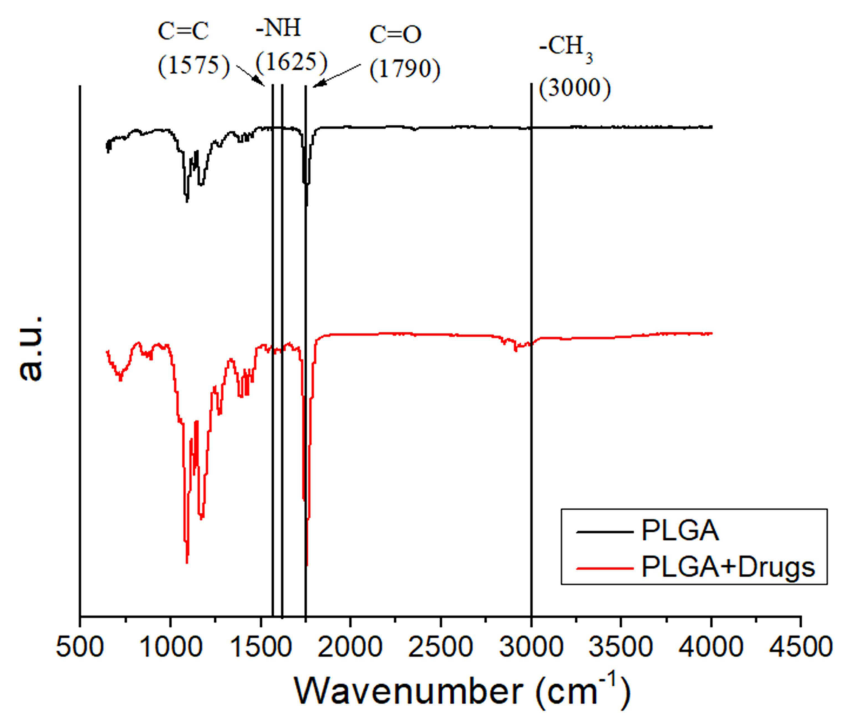

Figure 3 FTIR spectra of electrospun pure PLGA and drugs/hEGF-loaded PLGA nanofibers.

biocompatible, possesses a wide range of resorption times, and exhibits adjustable mechanical properties. In particular, PLGA has been extensively investigated for the development of devices for the controlled delivery of small molecule drugs, proteins, and other macromolecules. ${ }^{27,28}$

Vancomycin is a tricyclic glycopeptide antibiotic produced by Streptococcus orientalis. It is indicated in severe infections caused by Gram-positive bacteria, especially with the advent of methicillin-resistant Staphylococcus aureus and penicillin-

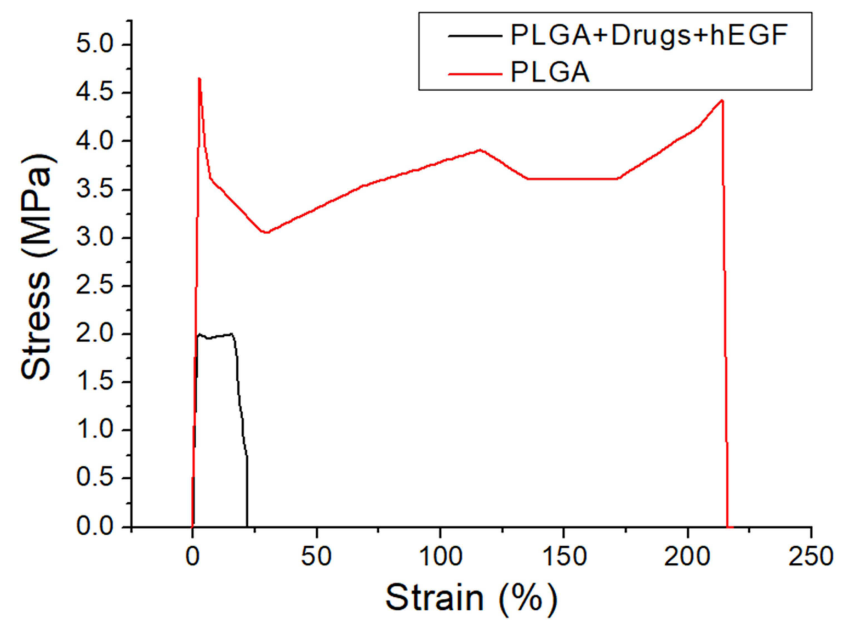

Figure 5 Stress-strain curve of pure PLGA and drugs/hEGF-loaded sandwichstructured nanofibers.

resistant pneumococci. Furthermore, vancomycin is also indicated in the treatment of patients allergic to penicillin and cephalosporins. ${ }^{29}$ Ceftazidime is a third-generation cephalosporin that possesses a broad spectrum of in vitro activity against Gram-positive and Gram-negative aerobic bacteria. The drug has been indicated for the treatment of lower respiratory tract infections, skin and skin structure infections, urinary tract infections, bacterial septicemia, bone and joint infections, gynecologic infections, intra-abdominal infections (including peritonitis), and central nervous system infections (including meningitis) caused by susceptible bacteria. ${ }^{30}$ Ketorolac is a nonsteroidal anti-inflammatory drug (NSAID) that has been widely employed for the short-term treatment of moderate to severe pain in adults. It works by blocking the body's production of certain natural substances that cause inflammation, and it is usually used before or after medical procedures or after surgery to decrease swelling, pain, or fever. ${ }^{31}$ Meanwhile, the epidermal growth factor (EGF) is a common mitogenic factor that stimulates the proliferation of different cell types, especially fibroblasts and epithelial cells. EGF activates the EGF receptor (EGFR/ErbB), which in turn initiates intracellular signaling. ${ }^{32-34}$

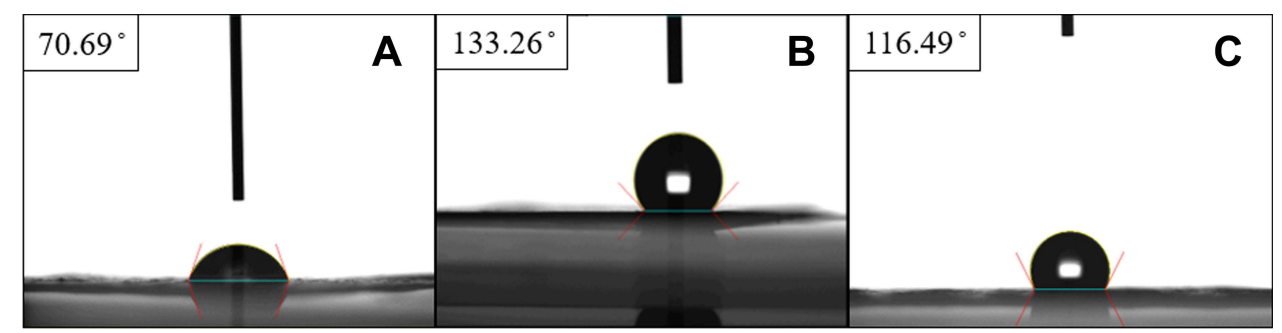

Figure 4 Measured contact angles. (A) PLGA/ketorolac nanofibers, (B) sheath-core structured PLGA/hEGF nanofibers, (C) PLGA/vancomycin/ceftazidime nanofibers. 
A

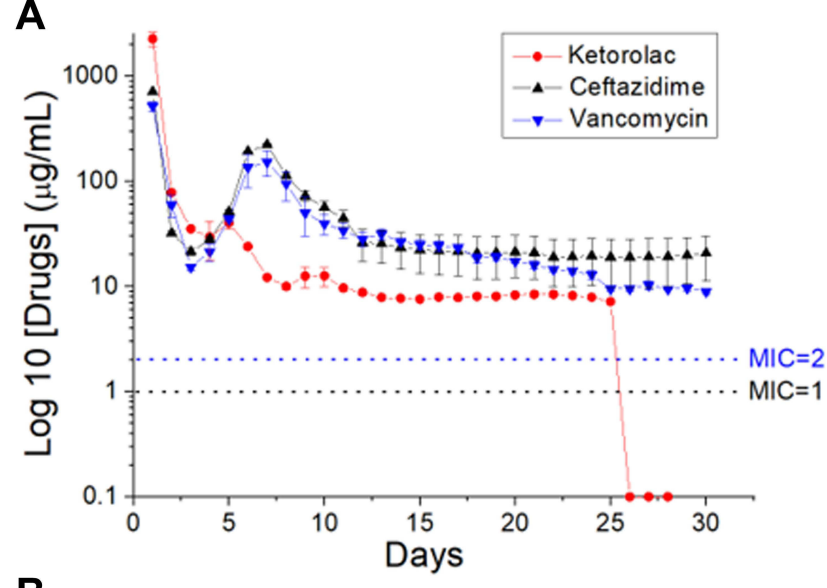

B

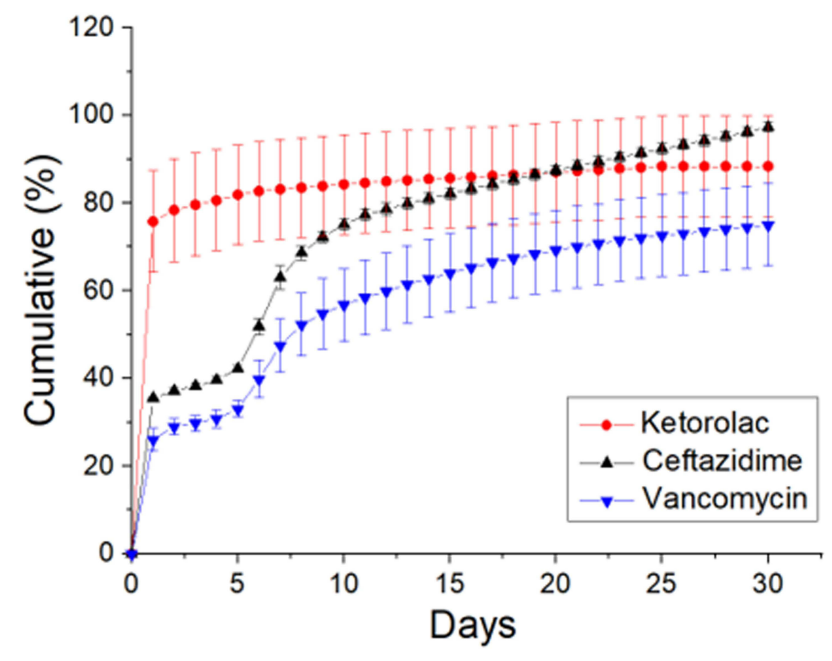

Figure 6 In vitro liberation patterns of ketorolac, vancomycin, and ceftazidime from the nanofibrous membranes.

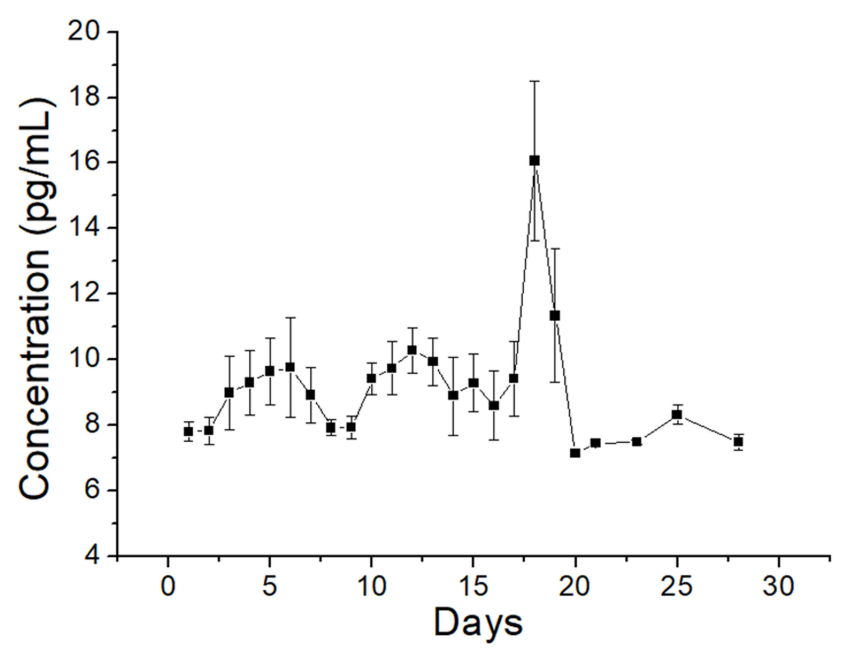

A
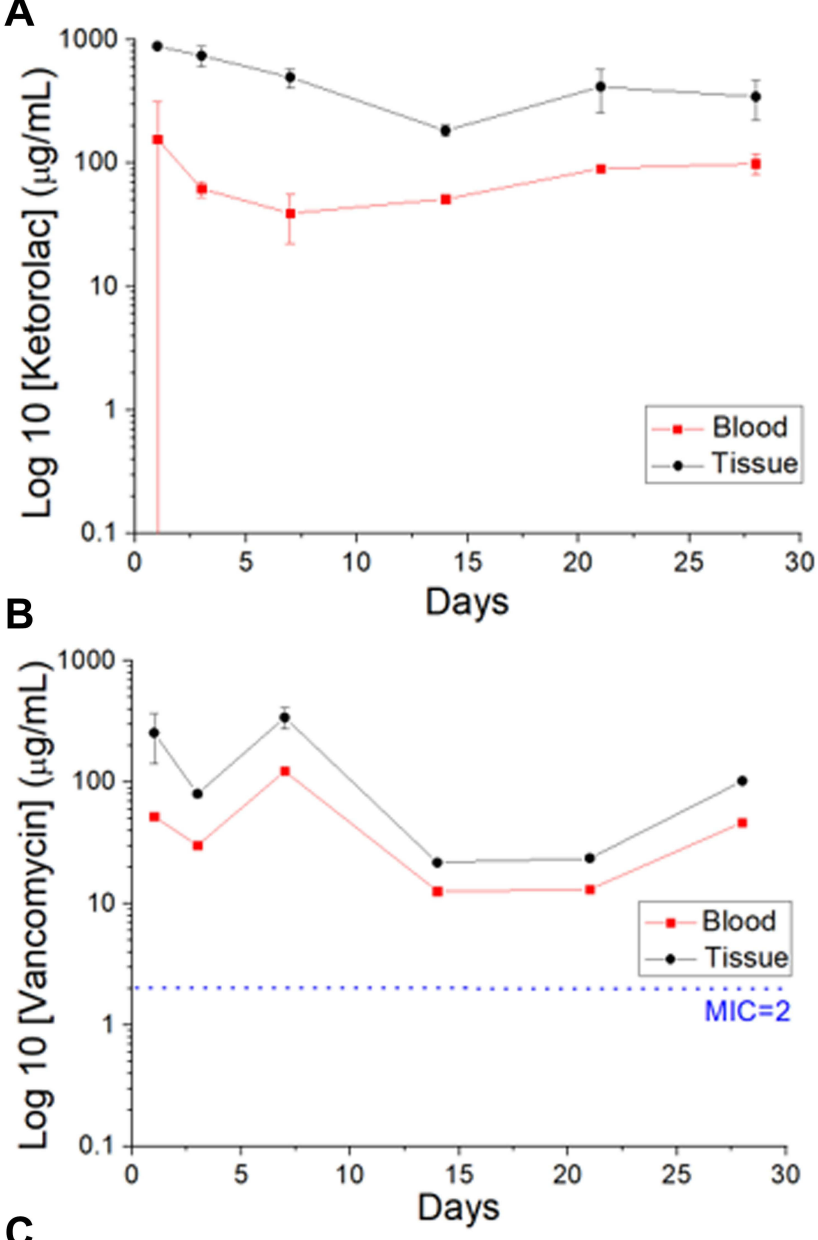

C

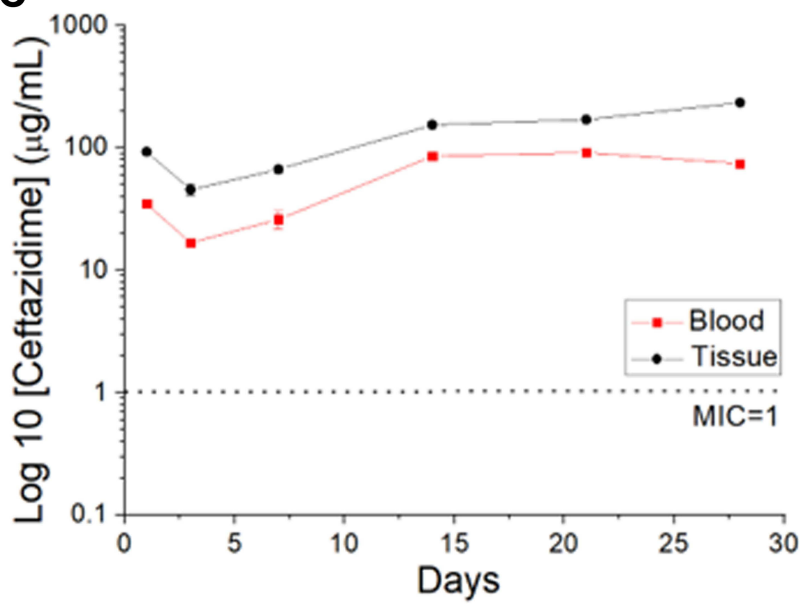

Figure 8 (A) Implantation of nanofibrous membrane, (B) no tissue adhesion was observed 14 days post-implantation, (C) tissue adhesion was noted in the control group (with no membrane).

Figure 7 In vitro elution profile of hEGF from the nanofibers. 


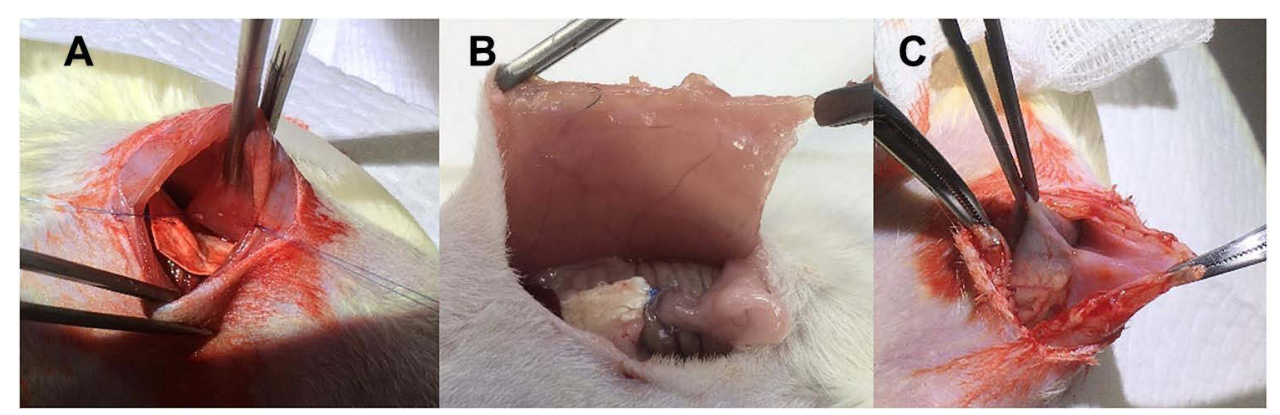

Figure 9 In vivo liberation of (A) ketorolac, (B) vancomycin, and (C) ceftazidime from the sandwich-structured nanofibers.

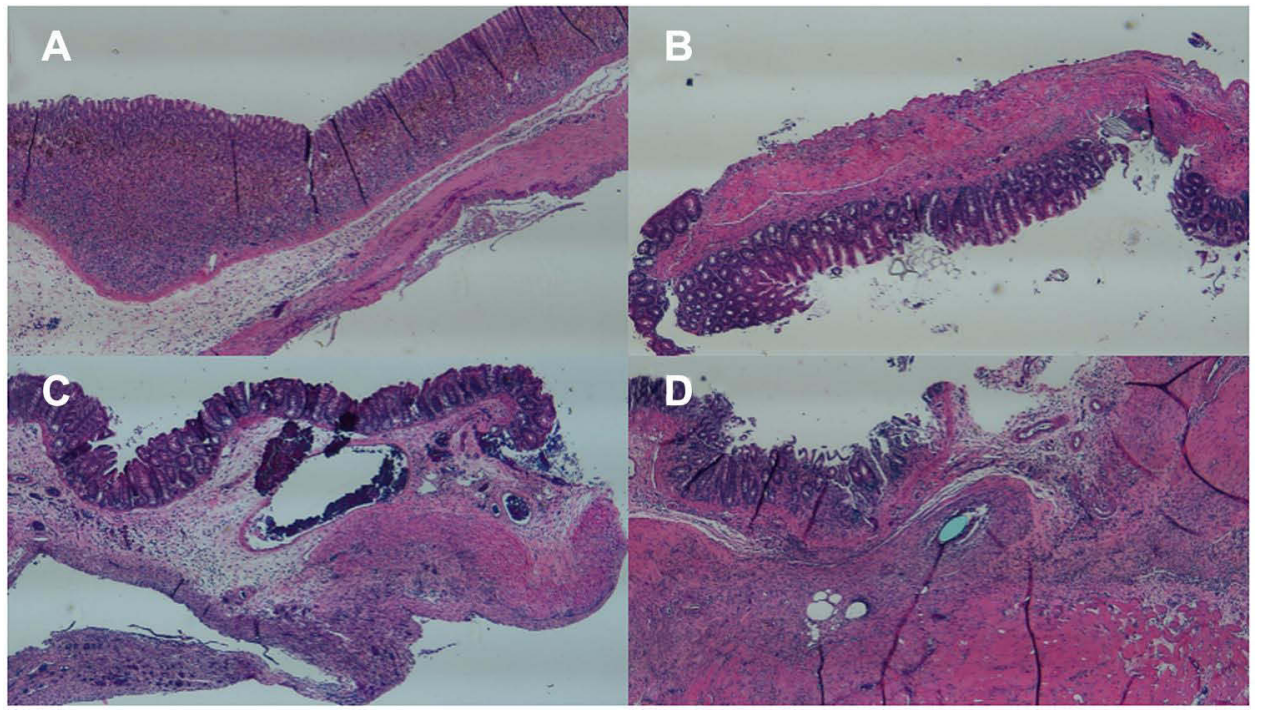

Figure 10 Histological images of the experimental group (with membranes) on (A) day I, (B) day 3, and (C) day 7, and the control group (without membranes) on (D) day 7 (Scale bar: $1 \mathrm{~mm}$ ).

Electrospinning and co-axial electrospinning are economical and effective methods for manufacturing regular and sheath/core-structured nanofibers. Electrospun nanofibrous membranes with drugs embedded in a PLGA matrix can provide controllable and sustainable drug release. Additionally, the nanofibers feature a large surface area and three-dimensional nanofibrous networks, thus mimicking native extracellular matrices. All these characteristics endow the nanofibrous membranes with tremendous potential for drug delivery and tissue engineering applications. Pharmaceuticals, various biomolecules, and many other agents can be satisfactorily incorporated into coaxially spun nanofibers for drug transport. The greatest advantage of the core-sheath layout is that the embedded agents remain active after the spinning procedure, mainly because of the protection from the sheath polymer.

Wound healing involves tissue repair in response to injury. It is a sequence of biological developments that start with hemostasis and later consist of inflammatory reactions. ${ }^{35}$ This involves the generation of connective tissues, enveloping of the wounds by the epithelium, and wound remodeling. Effective healing is dependent on growth factors and cytokine signals that promote keratinocyte de-differentiation and movement for reepithelialization. EGF is a predominant signaling molecule that activates epithelial cells' movement for reepithelialization. The molecule further serves as a fundamental stimulator of fibroblast migration and wound contraction. The hEGF-incorporated nanofibers can imitate an ideal EGF-eluting scaffold for advancing surgical wound healing.

Postoperative pain management is a precedency for patients. Inappropriately addressed postoperative pain may lead to complications and extended rehabilitation. Uncontrolled acute pain can lead to the formation of chronic pain, which limits the quality of life. Appropriate 
pain relief shortens hospitalization, reduces therapy costs, and improves patient satisfaction. Additionally, favorable pain management assists in speeding up wound healing and diminishes the risk of developing post-surgical complications, such as pneumonia and hypercoagulopathy.

Meanwhile, surgery involving an incision in the skin may result in postoperative wound infection. Antibiotics are generally used to prevent and treat wound infections. An adequate antimicrobial agent should be effective against microorganisms anticipated to incite infection, attain proper local tissue levels, and initiate minimal side effects.

Drug liberation from bioresorbable devices generally consists of three distinct phases: a burst, a diffusiongoverned phase, and a degradation-dominated phase. In the electrospinning procedure, most drugs are disbursed in the bulk of the polymeric frame. Nonetheless, certain drugs may be allocated on the surface of nanofibrous membranes, resulting in an initial burst. Post-burst, the liberation of pharmaceuticals is controlled and influenced by diffusion. A second peak elution was noted at day seven, after which the concentration gradually decreased. The results indicated that the electrospun polymeric drug-loaded membranes discharged high concentrations of vancomycin and ceftazidime (well above the minimum inhibitory concentration [MIC]) in vitro for over 30 days and ketorolac in vitro for over 25 days (Figure 6). Contrarily, a delicate and continuous release of hEGF from the core resulted from the sheltering effect of the sheath layer, leading to a sustainable elution of significant concentrations of hEGF for more than 28 days (Figure 7). Additionally, the experimental results in Figure 8 also suggested that the nanofibers eluted significant levels of the pharmaceuticals at the target region for over 28 days, while the drug levels remained much lower systemically. This provides the advantage of optimized surgical wound therapy with minimal systemic side effects. The multi-drugs/hEGF loaded sandwichstructured nanofibers showed their capacity to provide sustained release of antibiotics, analgesics, and growth factors at the wound site, thus facilitating post-surgery infection control, prolonged pain relief, and enhanced wound healing. Furthermore, the membranes demonstrated their efficacy as anti-adhesion agents.

Despite the primary discoveries, the current work has limitations. The first limitation is the relatively small number of studied animals. The other is that the transferability of the findings in this experimental study to humans remains unconfirmed. These will be topics for future studies.

\section{Conclusion}

In this study, we investigated the use of degradable multidrugs/hEGF sandwich-structured anti-adhesive nanofibers. The experimental outcomes indicate that degradable antiadhesive membranes discharged high levels of vancomycin/ceftazidime, ketorolac, and hEGF in vitro for more than 30, 24, and 27 days, respectively. The in vivo assessment in a rat laparotomy model indicated no adhesion in the peritoneal cavity at 14 days post-operation, demonstrating the anti-adhesive capability of the sandwichstructured nanofibrous membranes. The nanofibers also released effective levels of vancomycin, ceftazidime, and ketorolac for more than 28 days in vivo. The in vivo assessment in a rat laparotomy model indicated no adhesion in the peritoneal cavity at 14 days post-operation, demonstrating the anti-adhesive capability of the sandwich-structured nanofibrous membranes. Histological examination revealed no adverse effects. The nanofibrous membranes would provide advantages in post-surgery infection control, prolonged pain relief, and enhanced wound healing.

\section{Funding}

This work was financially sponsored by the Ministry of Science and Technology, Taiwan (Contract No. 110-2622E-182-005) and Chang Gung Memorial Hospital (Contract No. CRRPD2K0011 and CRRPD2K0021).

\section{Disclosure}

The authors report no conflicts of interest in this work.

\section{References}

1. Tang J, Xiang Z, Bernards MT, Chen S. Peritoneal adhesions: occurrence, prevention and experimental models. Acta Biomater. 2020;116:84-104.

2. Hellebrekers BWJ, Trimbos-Kemper GCM, van Blitterswijk CA, Bakkum EA, Trimbos JBMZ. Effects of five different barrier materials on post-surgical adhesion formation in the rat. Hum Reprod. 2000;15 (6):1358-1363. doi:10.1093/humrep/15.6.1358

3. Niwa D, Koide M, Fujie T, Goda N, Takeoka S. Application of nanosheets as an anti-adhesion barrier in partial hepatectomy. J Biomed Mater Res B. 2013;101(7):1151-1258. doi:10.1002/jbm. b. 32937

4. Bae SH, Son SR, Kumar SS, et al. Evaluation of the potential anti-adhesion effect of the PVA/Gelatin membrane. J Biomed Mater Res B. 2014;102(4):840-849. doi:10.1002/jbm.b.33066

5. Lo H-Y, Kuo H-T, Huang -Y-Y. Application of polycaprolactone as an anti-adhesion biomaterials film. Artif Organs. 2010;34(8):648-653. doi:10.1111/j.1525-1594.2009.00949.x

6. Lee M-W, Tsai H-F, Wen S-M, Huang C-H. Photocrosslinkable gellan gum film as an anti-adhesion barrier. Carbohydr Polym. 2012;90:1132-1138. doi:10.1016/j.carbpol.2012.06.064 
7. Liu S, Zhao J, Ruan H, et al. Antibacterial and anti-adhesion effects of the silver nanoparticles-loaded poly(L-lactide) fibrous membrane. Mater Sci Eng C. 2013;33:1176-1182. doi:10.1016/j. msec.2012.12.008

8. Lim JI, Kang MJ, Lee W-K. Lotus-leaf-like structured chitosanpolyvinyl pyrrolidone films as an anti-adhesion barrier. Appl Surf Sci. 2014;320:614-619. doi:10.1016/j.apsusc.2014.09.087

9. Sotiri I, Overton JC, Waterhouse A, Howell C. Immobilized liquid layers: a new approach to anti-adhesion surfaces for medical applications. Exp Biol Med. 2016;241:909-918. doi:10.1177/ 1535370216640942

10. Schnuriger B, Barmparas G, Branco BC, Lustenberfer T, Inaba K, Demetriades D. Prevention of postoperative peritoneal adhesions: a review of the literature. Amer J Surg. 2011;201(1):111-121.

11. Andersen BM. Prevention of postoperative wound infections. Prev Contr Infect Hosp. 2018;25:377-437.

12. Torpy JM, Burke AE, Glass RM. Postoperative infections. JAMA. 2010;303(24):2544. doi:10.1001/jama.303.24.2544

13. Neil MJE, Macrae WA. Post surgical pain: the transition from acute to chronic pain. Rev Pain. 2009;3(2):6-9. doi:10.1177/ 204946370900300203

14. Shoar S, Esmaeili S, Safari S. Pain management after surgery: a brief review. Anesth Pain Med. 2012;1(3):184-186. doi:10.5812/ kowsar.22287523.3443

15. Garimella V, Cellini C. Postoperative pain control. Clin Colon Rectal Surg. 2013;26(3):191-196. doi:10.1055/s-0033-1351138

16. Chen S, Wang G, Wu T, et al. Silver nanoparticles/ibuprofen-loaded poly(L-lactide) fibrous membrane: anti-infection and anti-adhesion effects. Int J Mol Sci. 2014;15(8):14014-14025. doi:10.3390/ ijms 150814014

17. Kao CW, Lee D, Wu MH, Chen JK, He HL, Liu SJ. Lidocaine/ ketorolac-loaded biodegradable nanofibrous anti-adhesive membranes that offer sustained pain relief for surgical wounds. Inter J Nanomed. 2017;12:5893-5901. doi:10.2147/IJN.S140825

18. Kao CW, Tseng YY, Liu KS, et al. Anesthetics and human epidermal growth factor incorporated into anti-adhesive nanofibers provide sustained pain relief and promote healing of surgical wounds. Inter J Nanomed. 2019;14:4007-4016. doi:10.2147/IJN.S202402

19. Hussein KH, Abdelhamid HN, Zou X, Woo H-M. Ultrasonicated graphene oxide enhances bone and skin wound regeneration. Mater Sci Eng C. 2019;94(1):484-492. doi:10.1016/j.msec.2018.09.051

20. Khan MS, Abdelhamid HN, Wu H-F. Near infrared (NIR) laser mediated surface activation of graphene oxide nanoflakes for efficient antibacterial, antifungal and wound healing treatment. Colloids Surf B. 2015;127(1):281-291. doi:10.1016/j.colsurfb.2014.12.049

21. Dinarvand P, Hashemi SM, Seyedjafari S, et al. Function of poly (lactic-co-glycolic acid) nanofiber in reduction of adhesion bands. J Surg Res. 2012;172:e1-e9. doi:10.1016/j.jss.2011.10.014
22. Zhang M, Li Z, Liu L, et al. Preparation and Characterization of Vancomycin-Loaded Electrospun Rana chensinensis Skin Collagen/ Poly(L-lactide) Nanofibers for Drug Delivery. $J$ Nanomat. 2016;2016:9159364. doi:10.1155/2016/9159364

23. Huang Y, Zhang Y, Yan Z, Liao S. Assay of ceftazidime and cefepime based on fluorescence quenching of carbon quantum dots. Luminescence. 2015;30(7):1133-1138. doi:10.1002/bio.2871

24. Gupta AK, Madan S, Majumdar DK, Maitra A. Ketorolac entrapped in polymeric micelles: preparation, characterization and ocular anti-inflammatory studies. Int $J$ Pharm. 2000;209(1-2):1-14. doi:10.1016/S0378-5173(00)00508-1

25. Nemati S, Kim SJ, Shin YM, Shin H. Current progress in application of polymeric nanofibers to tissue engineering. Nano Convergence. 2019;6:36. doi:10.1186/s40580-019-0209-y

26. Liu Z, Ramakrishna S, Liu X. Electrospinning and emerging healthcare and medicine possibilities. APL Bioeng. 2020;4(3):030901. doi:10.1063/5.0012309

27. Makadia HK, Siegel SJ. Poly Lactic-co-Glycolic acid (PLGA) as biodegradable controlled drug delivery carrier. Polymers (Basel). 2011;3(3):1377-1397. doi:10.3390/polym3031377

28. Hines DJ, Kaplan DL. Poly (lactic-co-glycolic acid) controlled release systems: experimental and modeling insights. Crit Rev Ther Drug Carrier Syst. 2013;30(3):257-276. doi:10.1615/ CritRevTherDrugCarrierSyst.2013006475

29. Bruniera FR, Ferreira FM, Saviolli LRM, et al. The use of vancomycin with its therapeutic and adverse effects: a review. Eur Rev Med Pharmacol Sci. 2015;19(4):694-700.

30. Richards DM, Brogden RN. Ceftazidime. A review of its antibacterial activity, pharmacokinetic properties and therapeutic use. Drugs. 1985;29(2):105-161. doi:10.2165/00003495-198529020-00002

31. Richards DM, Brogden RN. Ketorolac. A review of its pharmacodynamic and pharmacokinetic properties, and therapeutic potential. Drugs. 1990;39(1):86-109. doi:10.2165/00003495-199039010-00008

32. Wong RW, Guillaud L. The role of epidermal growth factor and its receptors in mammalian CNS. Cytokine Growth Factor Rev. 2004;15 (2-3):147-156. doi:10.1016/j.cytogfr.2004.01.004

33. Steed DL. The role of growth factors in wound healing. Surg Clin N Amer. 1997;77(3):575-586. doi:10.1016/S0039-6109(05)70569-7

34. Bodnar RJ. Epidermal growth factor and epidermal growth factor receptor: the Yin and Yang in the treatment of cutaneous wounds and cancer. Adv Wound Care. 2013;2(1):24-29. doi:10.1089/ wound.2011.0326

35. de Oliveira Gonzalez AC, Costa TF, de Araújo Andrade Z, Medrado ARAP. Wound healing-A literature review. An Bras Dermatol. 2016;91(5):614-620. doi:10.1590/abd18064841.20164741
International Journal of Nanomedicine

\section{Publish your work in this journal}

The International Journal of Nanomedicine is an international, peerreviewed journal focusing on the application of nanotechnology in diagnostics, therapeutics, and drug delivery systems throughout the biomedical field. This journal is indexed on PubMed Central, MedLine, CAS, SciSearch ${ }^{\mathbb{B}}$, Current Contents ${ }^{\mathbb{B}} /$ Clinical Medicine, $^{2}$
Journal Citation Reports/Science Edition, EMBase, Scopus and the Elsevier Bibliographic databases. The manuscript management system is completely online and includes a very quick and fair peer-review system, which is all easy to use. Visit http://www.dovepress.com/ testimonials.php to read real quotes from published authors. 